\title{
Expression of the MexXY-OprM efflux system in Pseudomonas aeruginosa with discordant cefepime/ceftazidime susceptibility profiles
}

\author{
Somvadee Laohavaleeson' \\ Karen Lolans 2,3 $^{2,3}$ \\ John P Quinn ${ }^{2,3,4}$ \\ Joseph L Kuti' \\ David P Nicolau' \\ 'Center for Anti-Infective Research \\ and Development, Hartford Hospital, \\ Hartford, CT, USA; ' John Stroger \\ Hospital, Chicago, IL, USA; ${ }^{3}$ Chicago \\ Infectious Disease Research Institute, \\ Chicago, IL, USA; ${ }^{4}$ Rush University \\ Medical Center, Chicago, IL, USA
}

\begin{abstract}
While MIC distributions and percent susceptibility for cefepime and ceftazidime are generally similar among Pseudomonas aeruginosa, we noted an increasing discordance in susceptibility favoring ceftazidime at our hospital. Quantitative reverse transcriptase-polymerase chain reaction was utilized to explore overexpression of the MexXY-OprM efflux as the mechanism for this phenotype profile. Thirteen of 15 (87\%) randomly selected isolates had mex $Y$ gene expression levels of 5.8-40.8-fold relative to the wild-type reference strain. While mex $Y$ overexpression was noted in the majority of isolates, other resistance mechanisms appear to contribute to the observed phenotypic profile of the Pseudomonas aeruginosa studied. Clinicians must understand not only the magnitude of difference in the MIC profiles between agents, but also the mechanism(s) responsible for these observations if strategies (ie, pharmacodynamic dosing) are to be designed to optimize patient care outcomes in the face of increasing resistance.
\end{abstract}

Keywords: Pseudomonas aeruginosa, efflux, MexXY-OprM, mexY, cefepime, resistance

\section{Introduction}

Pseudomonas aeruginosa is a commonly occurring nosocomial Gram-negative pathogen that is often associated with antibiotic resistant phenotypes, a profile that has been linked to therapeutic failure (Georges et al 2006; Mesaros et al 2007). This organism can confer multiple mechanisms of resistance, including $\beta$-lactamase production, expression of efflux pumps, and reduction of its outer-membrane (porin) permeability (Bonomo and Szabo 2006; Poole 2007; Tenover 2006). While resistance due to these mechanisms may be expressed concurrently, the presence of efflux pumps are an important singular determinant of multi-drug resistance (Bonomo and Szabo 2006; Dubois et al 2001; Nikaido and Zgurskaya 1999; Tenover 2006a; Tenover 2006b). There exist in $P$. aeruginosa naturally occurring multidrug-efflux (Mex) pumps that consist of three components: 1) a cytoplasmic-membrane associated the resistance-nodulation-division (RND) transmembrane protein transporter (eg, MexB, MexY), 2) an outer membrane gated channel (eg, OprM, OprN, OprJ), and 3) a periplasmic membrane fusion lipoprotein or pump assembly (eg, MexA, MexX) linking the two membrane bound components (Jeannot et al 2005). Four predominant Mex efflux systems have been reported: MexAB-OprM, MexCD-OprJ, MexEF-OprN, and MexXY-OprM (Aeschlimann 2003; Nikaido and Zgurskaya 1999; Schweizer 2003; Tenover 2006); each has a preferential set of antimicrobial agent substrates. For instance, MexXY-OprM is capable of extruding cefepime, cefotaxime, levofloxacin, ciprofloxacin, amikacin, gentamicin, tobramycin, erythromycin, tetracycline, and meropenem (Aeschlimann 2003; Masuda et al 2000; Pournaras et al 2005). The overexpression of MexXY in $P$. aeruginosa isolates was initially reported by 
Westbrock-Wadman and colleagues (1999) and then by Wolter and colleagues (2004) as the mechanism responsible for fluoroquinolone and aminoglycosides resistance, respectively.

Cefepime and ceftazidime are two cephalosporins used clinically against $P$. aeruginosa. Minimum inhibitory concentrations (MICs) and percent susceptibility for these agents are generally similar (Flamm et al 2004; Karlowsky et al 2005); however, in 2006, Hocquet et al reported the overexpression of the MexXY-OprM efflux pump as a major mechanism for $P$. aeruginosa isolates that were cefepime resistant/ceftazidime susceptible in two French hospitals (Hocquet et al 2006). The $\mathrm{MIC}_{50}$ for cefepime was 2 -fold higher than ceftazidime in this phenotype. While cefepime is a substrate for MexXY-OprM, ceftazidime does not appear to be affected by this type of efflux pump (Aeschlimann 2003).

At our 810 bed inner city teaching hospital, cefepime is widely used for empiric and definitive therapy of $P$. aeruginos $a$ After the formulary replacement of ceftazidime with cefepime in 2000, $P$. aeruginosa susceptibility to cefepime appeared to decline while the percent susceptible to ceftazidime remained stable over the next several years. Initially, we attributed this to errors in automated testing systems since it had been previously reported that some systems over report resistance for cefepime (Biedenbach et al 1999; Jones et al 1998; Sader et al 2006). Recently, however, we have detected $P$. aeruginosa strains that appear to be resistant to all $\beta$-lactams except ceftazidime. Upon further evaluation, these recent isolates were susceptible or intermediate to ceftazidime, non-susceptible to cefepime, and had cefepime MICs that were at least 2-fold greater than ceftazidime. As a result of these observations we sought to determine if one potential explanation for this phenotype profile was the overexpression of the MexXY-OprM efflux pump.

\section{Materials and methods}

$P$. aeruginosa clinical isolates collected at Hartford Hospital during an institutional survey in 2005-2006 were selected for analysis. We randomly chose 15 nonduplicate $P$. aeruginosa that were susceptible or intermediate to ceftazidime, nonsusceptible to cefepime, and had cefepime MICs that were at least 2-fold greater than that of ceftazidime. Most clinical isolates ( $n=9)$ were from respiratory specimens; 6 of the 9 were obtained from patients diagnosed with ventilator-associated pneumonia. The remaining isolates were collected from the urinary tract and wound samples. Cefepime and ceftazidime MICs were determined by E-Test ${ }^{\circledR}$ (AB Biodisk, Solna, Sweden) in duplicate and the mean values are reported.

\section{Pulsed-field gel electrophoresis}

Clonality was assessed by means of pulsed-field gel electrophoresis (PFGE). Chromosomal DNA was prepared by the method of Matushek et al (Matushek et al 1996), digested with XbaI, and separated by electrophoresis on a CHEF Mapper apparatus (BioRad Laboratories, Richmond, CA) at a voltage of $6 \mathrm{~V} / \mathrm{cm}$ at $14{ }^{\circ} \mathrm{C}$ for $21 \mathrm{~h}$, with pulse times linearly ramped from 1 to $25 \mathrm{~s}$. Strain relatedness was determined according to the criteria by Tenover et al (Tenover et al 1995).

\section{Isolation of total RNA}

Strains were inoculated in $2 \mathrm{ml}$ calcium-adjusted Mueller Hinton broth (CaMHB) and grown overnight at $35^{\circ} \mathrm{C}$. Strains were diluted 1:1000 in CaMHB and cultures grown to an absorbance at $600 \mathrm{~nm}$ of 0.3 (corresponding to logarithmic phase). A $1.8 \mathrm{ml}$ aliquot of this culture was added to $3.6 \mathrm{ml}$ of RNA easy bacteria protect solution (Qiagen, Valencia, CA) and total RNA was isolated using the Qiagen RNeasy kit (Qiagen). Residual DNA was eliminated by an oncolumn DNAse digestion using the RNAse-free DNase set (Qiagen), and the RNA was resuspended in $30 \mu \mathrm{l}$ of RNAse-free $\mathrm{H}_{2} \mathrm{O}$.

\section{Synthesis of cDNA for reverse transcriptase-polymerase chain reaction (RT-PCR)}

Reverse transcription to cDNA was performed using the Transcriptor First Strand cDNA Synthesis Kit (Roche, Indianapolis, IN) with random hexamers $(60 \mu \mathrm{M})$ as described in depth by the manufacturer's instructions. The initial reaction mixture contained $2 \mu \mathrm{g}$ of total RNA, which was subsequently divided into two aliquots. Reverse transcriptase was added to one aliquot, while the other aliquot served as a negative control to detect any residual genomic DNA in the RNA preparation. The cDNAs were stored at $-20^{\circ} \mathrm{C}$ until further analyses.

\section{RT-PCR analysis}

Expression of the MexXY-OprM operon was assessed by quantitative RT-PCR with specific primers to determine the transcript levels of the gene mexY (Hocquet et al 2006) as previously described. A LightCycler ${ }^{\circledR} 2.0$ RT-PCR machine (Roche, software version 3.5) was used for the quantification of cDNA. Duplicate PCR reactions were performed using

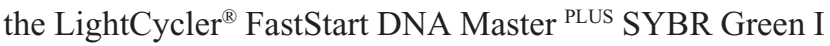
kit (Roche) according to manufacturer's instructions. A final concentration of $10 \%$ DMSO and 100 ng cDNA was used 
in a total volume of $20 \mu \mathrm{l}$ for each reaction. After a $10 \mathrm{~min}$ activation of the Taq polymerase at $95^{\circ} \mathrm{C}, 40$ cycles of $15 \mathrm{~s}$ at $95{ }^{\circ} \mathrm{C}, 5 \mathrm{~s}$ at $62{ }^{\circ} \mathrm{C}, 12 \mathrm{~s}$ at $72{ }^{\circ} \mathrm{C}$ were performed. A melt curve was run at the end of 40 cycles to test for the presence of a unique PCR reaction product.

The isogenic strains PAO1 (PAM1020, wild-type efflux expression) (Lomovskaya et al 1999) and PAM1323 (MexXY-OprM overexpressed) (gifts from O. Lomovskaya, Mpex Pharmaceuticals) served as controls and were analyzed in parallel with the clinical strains. The housekeeping gene, rpoD (sigma factor of RNA polymerase) (Savli et al 2003), served as the internal standard to correct for variations in the amount of starting template material. The threshold cycle $(\mathrm{Ct})$ of each sample, which identified the cycle number during PCR when fluorescence exceeded a threshold value, was determined by the software, and the relative expression of mex $Y$ was assessed using the $\Delta \Delta \mathrm{Ct}$ method, as described previously (Livak and Schmittgen 2001). The results represent relative expression levels (fold change) for a given target gene in the tested isolates compared to the PAO1 wild-type strain. Additionally, three clinical strains, having the same/similar MICs to both cefepime and ceftazidime, were used as efflux controls and showed mRNA transcript levels of mex $Y$ very close to that of PAO1 (0.8 times). The gain-of-efflux control strain demonstrated increased expression of mexY (26.2 times). A specific mRNA level of mexY greater than 4 times that of the wild-type susceptible strain PAO1 was considered to be a MexXY overproducer.

\section{Results}

After the introduction and exclusive use of cefepime in 2000, our $P$. aeruginosa susceptibility as noted by the institutional antibiogram has been relatively constant for ceftazidime but appeared to drop for cefepime (Table 1).
The cefepime/ceftazidime MIC ratio of the studied isolates ranged from 2.7 to $\geq 32$ (Table 2 ). As determined by PFGE, ten of the fifteen isolates clustered into three clonal groups. Group 1 consisted of the closely related isolates, PSA766 and 987 (2-band difference); Group 2 consisted of the indistinguishable PSA828, 882, and 782, and the closely-related PSA805 (2-band difference), and Group 3 consisted of the indistinguishable PSA814, 859, and 808, and the closely-related PSA855 (2-band difference). The five remaining isolates were unique pulsotypes.

In contrast to the dually susceptible control isolates, which showed no appreciable increase in mex $Y$ transcript levels, thirteen of the 15 isolates (87\%) exhibited a marked increased in mexY gene expression levels (from 5.8- to 40.8-fold) relative to the reference wild-type susceptible strain PAO1 (Table 2). The two clinical isolates that did not appear to show overexpression are designated as PSA784 and PSA831. While mexY overexpression was noted in the majority of these isolates, this was poorly correlated to the cefepime/ceftazidime MIC ratio. For example, while isolates PSA859 and PSA808 expressed high levels of $\operatorname{mexY}$ (21.3- and 34.3-fold higher than those observed in PAO1), their MIC ratios were 2.7 and 4.0, respectively. Moreover while PSA808 and PSA831 had the same cefepime/ceftazidime MIC ratio, PSA808 demonstrated high mexY expression (31.8-fold more than PAO1), while PSA831 did not.

Additionally, many of the MexY positive strains were multi-drug resistant. All 13 of these strains were resistant to ciprofloxacin and levofloxacin with the majority of MICs $>32 \mu \mathrm{g} / \mathrm{ml}$. Forty-six percent (6/13) were meropenem nonsusceptible (1 intermediate, 5 resistant) and 1/13 (8\%) was piperacillin-tazobactam resistant (MIC $>256 \mu \mathrm{g} / \mathrm{ml}$ ). Aminoglycoside MIC data were available for only 9 isolates, 8 of which were MexY positive: 5/8 were gentamicin resistant, $3 / 8$ were resistant to tobramycin.

Table I Hartford Hospital susceptibility for Pseudomonas aeruginosa to cefepime and ceftazidime from the year of cefepime formulary introduction to present

\begin{tabular}{lllll}
\hline Year Collected & \# isolates* & \multicolumn{2}{l}{ Susceptibility (\%) } & Difference (\%) \\
\cline { 3 - 4 } & & Cefepime & Ceftazidime & 4 \\
2000 & 945 & 77 & 81 & 13 \\
2001 & 1041 & 74 & 87 & 10 \\
2002 & 1022 & 75 & 85 & 8 \\
2003 & 900 & 74 & 82 & 9 \\
2004 & 1207 & 72 & 81 & 77 \\
2005 & 1400 & 65 & 82 & 12 \\
2006 & 1256 & 69 & & 13 \\
\hline
\end{tabular}

Note: *Isolates collected hospital-wide. 
Table 2 Results of MICs, PFGE, relative expression of mexY in Pseudomonas aeruginosa expressing the cefepime resistant/ceftazidime nonresistant phenotype

\begin{tabular}{|c|c|c|c|c|c|c|}
\hline \multirow[t]{2}{*}{ Strain ID } & \multirow[t]{2}{*}{ PFGE } & \multicolumn{2}{|c|}{ MIC $(\mu \mathrm{g} / \mathrm{ml})$} & \multirow[t]{2}{*}{ FEP/CAZ MIC ratio } & \multirow[t]{2}{*}{ Specific mRNA level ${ }^{\mathrm{b}}$} & \multirow[t]{2}{*}{ mexY Overexpression ${ }^{c}$} \\
\hline & & FEP & CAZ & & & \\
\hline Wild-type efflux strain PAOI & - & 1.5 & 1.25 & 1.20 & 1.0 & \\
\hline Susceptible clinical strains $(n=3)$ & - & $1.5-3$ & $1.5-3$ & $1.0-1.5$ & 0.8 & \\
\hline Gain-of-efflux strain PAM I 323 & - & 7 & 1.25 & 5.60 & 26.2 & + \\
\hline PSA 766 & NPSAI & 24 & 8 & 3.00 & 5.8 & + \\
\hline PSA 987 & NPSAIa & 32 & 8 & 4.00 & 5.9 & + \\
\hline PSA 828 & NPSA2 & 16 & 3 & 5.33 & 11.2 & + \\
\hline PSA 882 & NPSA2 & 32 & 6 & 5.33 & 7.0 & + \\
\hline PSA 782 & NPSA2 & 24 & 4 & 6.00 & 11.8 & + \\
\hline PSA 805 & NPSA2a & 24 & 6 & 4.00 & 6.8 & + \\
\hline PSA 814 & NPSA3 & 128 & 12 & 10.67 & 40.8 & + \\
\hline PSA 859 & NPSA3 & 32 & 8 & 4.00 & 21.3 & + \\
\hline PSA 808 & NPSA3 & 16 & 6 & 2.67 & 31.8 & + \\
\hline PSA 855 & NPSA3a & 24 & 6 & 4.00 & 22.8 & + \\
\hline PSA 820 & NPSA4 & 12 & 2 & 6.00 & 18.1 & + \\
\hline PSA $932^{d}$ & NPSA5 & 512 & 16 & $\geq 32.00$ & 16.7 & + \\
\hline PSA 776 & NPSA6 & 16 & 4 & 4.00 & 34.3 & + \\
\hline PSA 784 & NPSA7 & 32 & 6 & 5.33 & 2.6 & - \\
\hline PSA 83I & NPSA8 & 16 & 6 & 2.67 & 0.7 & - \\
\hline
\end{tabular}

Abbreviations: CAZ, ceftazidime; FEP, cefepime; MIC, minimum inhibitory concentration; PFGE, pulsed-field gel electrophoresis.

Notes: alsolates with clonal relations were grouped and separated by thinner line; 'b Specific mRNAs were quantified by real-time PCR after retrotranscription. Values are means of two independent determinations and are expressed relative to PAOI, which was set at I.0; ' $m$ ex $Y$ overexpression is indicated by a + sign; ${ }^{\mathrm{b}}$ Experimentally determined cefepime MIC $>256 \mu \mathrm{g} / \mathrm{ml}$; for calculation purposes, the value of $5 \mathrm{I} 2$ was utilized.

Together these data suggest that while the overexpression of the MexXY-OprM efflux pump may play an important role in our noted cefepime and ceftazidime MIC discordance, other currently unidentified mechanisms appear are contribute this phenotype profile.

\section{Discussion}

To our knowledge, these are the first US derived data set to suggest that the MexXY-OprM efflux pump is a contributory factor for the observed discordant susceptibility profile between cefepime and ceftazidime in $P$. aeruginosa. Our results also confirm that the discordant MICs observed between cefepime and ceftazidime are not simply attributable to errors in the automated susceptibility systems but in fact, occur at least in part from mexY gene overexpression.

The majority of the $P$. aeruginosa isolates analyzed in this study over expressed the MexXY-OprM efflux pump. The degree of mex $Y$ gene overexpression and variability was similar between our study (5.8-40.8-fold) and that of Hocquet and colleagues (2006) (4.0-39.0-fold). Some of our clinical isolates also displayed resistance to meropenem, ciprofloxacin, levofloxacin, gentamicin and tobramycin, all of which are known to be MexXY-OprM substrates (Aeschlimann 2003; Masuda et al 2000; Pournaras et al 2005).

The poor correlation between the cefepime/ceftazidime MIC ratio and the overexpression of mex $Y$ may be explained by the presence of other resistance mechanisms (ie, $\beta$-lactamases, outer-membrane proteins and/or other types of efflux. While studies to fully elucidate other possible mechanisms were not undertaken, the production of a $\beta$-lactamase, OXA-31, has been reported to also result in a cefepime resistant/ceftazidime susceptible phenotype in $P$. aeruginosa (Aubert et al 2001). Double efflux pumps (MexAB-OprM and MexXY-OprM) have also been identified in some strains of $P$. aeruginosa that were resistant to meropenem and cefepime but susceptible to ceftazidime (Pournaras et al 2005).

Currently, the true prevalence of MexXY-OprM expressing $P$. aeruginosa is unknown at our hospital, so these data, along with declining cefepime susceptibilities have prompted us to empirically use higher cefepime dosages based on pharmacodynamics (eg, 2 g every 
8 hours as prolonged infusions) or choose alternative antibiotics (ie, piperacillin/tazobactam). Additionally, the use of ceftazidime as definitive therapy for ceftazidime susceptible/cefepime non-susceptible $P$. aeruginosa has been considered.

In conclusion, our observation of MIC discordance between cefepime and ceftazidime in $P$. aeruginos a appears in part to be due to the overexpression of the MexXY-OprM efflux pump. Additional studies are required to fully elucidate the mechanism(s) responsible for this atypical phenotypic profile in $P$. aeruginosa.

\section{Acknowledgments}

This work was supported in part by a grant from AstraZeneca Pharmaceuticals, Inc., Wilmington, DE.

\section{References}

Aeschlimann JR. 2003. The role of multidrug efflux pumps in the antibiotic resistance of Pseudomonas aeruginosa and other gram-negative bacteria. Insights from the Society of Infectious Diseases Pharmacists. Pharmacotherapy, 23:916-24.

Aubert D, Poire L, Chevalier J, et al. 2001. Oxacillinase-mediated resistance to cefepime and susceptibility to ceftazidime in Pseudomonas aeruginosa. Antimicrob Agents Chemother, 45:1615-20.

Biedenbach DJ, Marshall SA, Jones RN. 1999. Accuracy of cefepime antimicrobial susceptibility testing results for Pseudomonas aeruginosa tested on the MicroScan WalkAway System. Diagn Microbiol Infect Dis, 33:305-7.

Bonomo RA, Szabo D. 2006 Mechanisms of multidrug resistance in Acinetobacter species and Pseudomonas aeruginosa. Clin Infect Dis, 43(Suppl 2):S49-56.

Dubois V, Arpin C, Melon M, et al. 2001. Nosocomial outbreak due to a multiresistant strain of Pseudomonas aeruginosa P12: efficacy of cefepime-amikacin therapy and analysis of beta-lactam resistance. J Clin Microbiol, 39:2072-8.

Flamm RK, Weaver MK, Thornsberry C, et al. 2004. Factors associated with relative rates of antibiotic resistance in Pseudomonas aeruginosa isolates tested in clinical laboratories in the United States from 1999 to 2002. Antimicrob Agents Chemother, 48:2431-6.

Georges B, Conil JM, Dubouix A, et al. 2006. Risk of emergence of Pseudomonas aeruginosa resistance to beta-lactam antibiotics in intensive care units. Crit Care Med, 34:1636-41.

Hocquet D, Nordmann P, El Garch F, et al. 2006. Involvement of the MexXY-OprM efflux system in emergence of cefepime resistance in clinical strains of Pseudomonas aeruginosa. Antimicrob Agents Chemother, 50:1347-51.

Jeannot K, Sobel ML, El Garch F, et al. 2005. Induction of the MexXY efflux pump in Pseudomonas aeruginosa is dependent on drug-ribosome interaction. J Bacteriol, 187:5341-6.
Jones RN, Biedenbach DJ, Marshall SA, et al. 1998. Evaluation of the Vitek system to accurately test the susceptibility of Pseudomonas aeruginosa clinical isolates against cefepime. Diagn Microbiol Infect Dis, 32:107-10.

Karlowsky JA, Jones ME, Thornsberry C, et al. 2005. Stable antimicrobial susceptibility rates for clinical isolates of Pseudomonas aeruginosa from the 2001-2003 tracking resistance in the United States today surveillance studies. Clin Infect Dis, 40(Suppl 2):S89-98.

Livak KJ, Schmittgen TD. 2001. Analysis of relative gene expression data using real-time quantitative PCR and the 2(-Delta Delta C(T)) Method. Methods, 25:402-8.

Lomovskaya O, Lee A, Hoshino K, et al. 1999. Use of a genetic approach to evaluate the consequences of inhibition of efflux pumps in Pseudomonas aeruginosa. Antimicrob Agents Chemother, 43:1340-6.

Masuda N, Sakagawa E, Ohya S, et al. 2000. Contribution of the MexX-MexY-oprM efflux system to intrinsic resistance in Pseudomonas aeruginosa. Antimicrob Agents Chemother, 44:2242-6.

Matushek MG, Bonten MJ, Hayden MK. 1996. Rapid preparation of bacterial DNA for pulsed-field gel electrophoresis. J Clin Microbiol, $34: 2598-600$.

Mesaros N, Nordmann P, Plesiat P, et al. 2007. Pseudomonas aeruginosa: resistance and therapeutic options at the turn of the new millennium. Clin Microbiol Infect, 13:560-78.

Nikaido H, Zgurskaya HI. 1999. Antibiotic efflux mechanisms. Curr Opin Infect Dis, 12:529-36.

Poole K. 2007. Efflux pumps as antimicrobial resistance mechanisms. Ann Med, 39:162-76.

Pournaras S, Maniati M, Spanakis N, et al. 2005. Spread of efflux pump-overexpressing, non-metallo-beta-lactamase-producing, meropenemresistant but ceftazidime-susceptible Pseudomonas aeruginosa in a region with blaVIM endemicity. J Antimicrob Chemother, 56:761-4.

Sader HS, Fritsche TR, Jones RN. 2006. Accuracy of three automated systems (MicroScan WalkAway, VITEK, and VITEK 2) for susceptibility testing of Pseudomonas aeruginosa against five broad-spectrum beta-lactam agents. J Clin Microbiol, 44:1101-4.

Savli H, Karadenizli A, Kolayli F, et al. 2003. Expression stability of six housekeeping genes: A proposal for resistance gene quantification studies of Pseudomonas aeruginosa by real-time quantitative RT-PCR. J Med Microbiol, 52:403-8.

Schweizer HP. 2003. Efflux as a mechanism of resistance to antimicrobials in Pseudomonas aeruginosa and related bacteria: unanswered questions. Genet Mol Res, 2:48-62.

Tenover FC. 2006a. Mechanisms of antimicrobial resistance in bacteria. Am J Med, 119:S3-10; discussion S62-70.

Tenover FC. 2006b. Mechanisms of antimicrobial resistance in bacteria. Am J Infect Control, 34:S3-10; discussion S64-73.

Tenover FC, Arbeit RD, Goering RV, et al. 1995. Interpreting chromosomal DNA restriction patterns produced by pulsed-field gel electrophoresis: criteria for bacterial strain typing. J Clin Microbiol, 33:2233-9.

Westbrock-Wadman S, Sherman DR, Hickey MJ, et al. 1999. Characterization of a Pseudomonas aeruginosa efflux pump contributing to aminoglycoside impermeability. Antimicrob Agents Chemother, 43:2975-83

Wolter DJ, Smith-Moland E, Goering RV, et al. 2004. Multidrug resistance associated with mexXY expression in clinical isolates of Pseudomonas aeruginosa from a Texas hospital. Diagn Microbiol Infect Dis, 50:43-50. 
